\title{
Approximate Numerical Solutions for Linear Volterra Integral Equations Using Touchard Polynomials
}

\author{
Jalil Talab Abdullah
}

Department of Statistics, Faculty of Administration and Economics, Wasit University, Iraq.

E-mail: jalil.talab@gmail.com

ORCID ID: https://orcid.org/ 0000-0002-6847-3635

Received 29/7/2019, Accepted 30/1/2020, Published 1/12/2020

This work is licensed under a Creative Commons Attribution 4.0 International License.

\begin{abstract}
:
In this paper, Touchard polynomials (TPs) are presented for solving Linear Volterra integral equations of the second kind (LVIEs-2k) and the first kind (LVIEs-1k) besides, the singular kernel type of this equation. Illustrative examples show the efficiency of the presented method, and the approximate numerical (AN) solutions are compared with one another method in some examples. All calculations and graphs are performed by program MATLAB2018b.
\end{abstract}

Keywords: Approximate Numerical Solutions, Linear Volterra Integral Equation, Touchard Polynomials.

\section{Introduction:}

In many problems, the Volterra integral equations (VIEs) arise from the real life, such as population dynamics, feedback control theory, and fluid dynamics etc. (1). Furthermore, many applications of engineering or physics can often lead to Volterra integral equations. The singular status, which appears in the physical modelling structures, has importance in mathematical physics and other sciences. The weakly singular kernel of the (VIEs) represents such phenomena, which have important applications in mathematical physics, electrochemistry, chemical reactions, superfluidity, and heat conduction (2).

The standard forms of (LVIEs-2k) and (LVIEs-1k) $(3,4)$ respectively defined as follows:

$$
\begin{gathered}
\mathrm{A}(\zeta)=\mathrm{k}(\zeta)+\alpha \int_{\mathrm{a}}^{\zeta} \mathrm{G}(\zeta, \tau) \mathrm{A}(\tau) \mathrm{d} \tau \quad[\mathrm{a}, \zeta] \\
-\mathrm{k}(\zeta)=\alpha \int_{\mathrm{a}}^{\zeta} \mathrm{G}(\zeta, \tau) \mathrm{A}(\tau) \mathrm{d} \tau \quad, \quad[\mathrm{a}, \zeta] \\
\subseteq \mathrm{R}
\end{gathered}
$$

and the general form of Abel's singular (LVIEs-1k) (4) is:

$$
\mathrm{k}(\zeta)=\alpha \int_{\mathrm{a}}^{\zeta} \frac{1}{\sqrt{\zeta-\tau}} \mathrm{A}(\tau) \mathrm{d} \tau \quad, \quad[\mathrm{a}, \zeta]
$$

Which is derived from a concrete problem of physics without passing during differential equations (5). $A(\zeta)$ is an unknown function that must be calculated, $\alpha$ is a known constant, it takes physical meanings of the characteristic of the material, and $\mathrm{G}(\zeta, \tau)$ is the kernel of the Integral equation (IE), which is a known continuous or noncontinuous function ( the kernel of such equations has jump discontinuities along the continuous curve which started at the origin (6) ), holds characteristic and property of the material, $\mathrm{k}(\zeta)$ is the function of the integration surface which is a known.

There are many numerical methods used or developed by the researchers to obtain the approximate numerical solutions for the (LVIEs), some of them are mentioned as follows: (7) gave the numerical scheme for the approximation of the (LVIEs) with highly oscillatory Bessel kernels. (8) applied the optimal homotopy asymptotic method for finding the approximate solutions of a class of the (LVIEs) with weakly singular kernels. (9) applied the standard spectral Galerkin polynomial method and a variant to solve a weakly singular for the (VIEs). (10) extended the single-step pseudospectral method for the (VIEs-2k) to the multistep pseudo-spectral method. (11) used the Galerkin 
weight residual numerical method with Chebyshev polynomials and Touchard polynomials as a trial function to obtain a numerical solution for the (IEs).

In this work, Touchard polynomials method is used to solve (LVIEs) numerically. The rest of the paper is arranged as follows: The solution method, approximation function, algorithm of solution, accuracy of the solution, convergence rate, test illustrative examples and the corresponding Tables and Figs. are presented. Finally, brief conclusions and references are listed.

\section{Solution Method:}

The Touchard polynomials (11, 12, 13 and 14) were first studied by the French mathematician Jacques Touchard (1885-1968), it's a polynomial sequence of binomial type over the interval $[0,1]$, and have the form:

$\mathrm{Z}_{\mathrm{n}}(\zeta)=\sum_{\mathrm{r}=0}^{\mathrm{n}} \mathrm{S}(\mathrm{n}, \mathrm{r}) \zeta^{\mathrm{r}}=\sum_{\mathrm{r}=0}^{\mathrm{n}}\left(\begin{array}{l}\mathrm{n} \\ \mathrm{r}\end{array}\right) \zeta^{\mathrm{r}}$

Where $\left(\begin{array}{l}n \\ r\end{array}\right)=\frac{n !}{r !(n-r) !}, n$ is the degree of the polynomials, $r$ is the index of the polynomials.

The first seven polynomials of the (TPs) are given below:

$$
\begin{aligned}
& \mathrm{Z}_{0}(\zeta)=1 \\
& \mathrm{Z}_{1}(\zeta)=1+\zeta \\
& \mathrm{Z}_{2}(\zeta)=1+2 \zeta+\zeta^{2} \\
& \mathrm{Z}_{3}(\zeta)=1+3 \zeta+3 \zeta^{2}+\zeta^{3} \\
& \mathrm{Z}_{4}(\zeta)=1+4 \zeta+6 \zeta^{2}+4 \zeta^{3}+\zeta^{4} \\
& \mathrm{Z}_{5}(\zeta)=1+5 \zeta+10 \zeta^{2}+10 \zeta^{3}+5 \zeta^{4}+\zeta^{5} \\
& \mathrm{Z}_{6}(\zeta)=1+6 \zeta+15 \zeta^{2}+20 \zeta^{3}+15 \zeta^{4}+6 \zeta^{5}+\zeta^{6}
\end{aligned}
$$$$
\text { Approximation Function: }
$$

For determining the approximate numerical solutions of Eq. (1), the function $A_{n}(\zeta)$ is approximated by the (TPs) is as follows:

$$
\begin{aligned}
A_{n}(\zeta)=\theta_{0} Z_{0}(\zeta) & +\theta_{1} Z_{1}(\zeta)+\cdots+\theta_{n} Z_{n}(\zeta) \\
= & \sum_{r=0}^{n} \theta_{r} Z_{r}(\zeta), \quad 0 \leq \zeta \\
\leq 1 & \cdots(3)
\end{aligned}
$$

where the function $\left\{\mathrm{Z}_{\mathrm{r}}(\zeta)\right\}_{\mathrm{r}=0}^{\mathrm{n}}$ denotes the Touchard basis polynomials of nth degrees, as defined in Eq.

(2). The $\theta_{\mathrm{r}}(\mathrm{r}=0,1, \ldots, \mathrm{n})$ are the unknown

Touchard coefficients that must be determined, and $\mathrm{n}$ represents any positive integer. Writing Eq. (3) as a dot product:

$A_{n}(\zeta)=\left[\begin{array}{lll}Z_{0}(\zeta) & Z_{1}(\zeta) \ldots Z_{n}(\zeta)\end{array}\right] \cdot\left[\begin{array}{c}\theta_{0} \\ \theta_{1} \\ \cdot \\ \cdot \\ \theta_{n}\end{array}\right]$,
$A_{n}(\zeta)=$

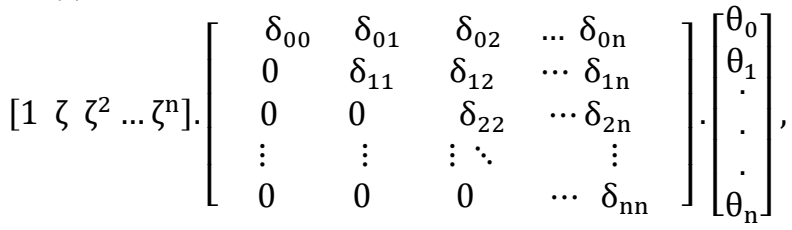

where $\delta_{\mathrm{kk}}(\mathrm{k}=0,1,2, \ldots, \mathrm{n})$ are known constants of the power basis, used to determine the (TPs), this matrix is upper triangular. For instance, in cases: $\mathrm{n}=2,3,4$ and 5 , the operational matrices will be shown in the Eq. (6), (6a), (6b) and (7) respectively:

$$
A_{2}(\zeta)=\left[\begin{array}{lll}
1 & \zeta & \zeta^{2}
\end{array}\right] \cdot\left[\begin{array}{lll}
1 & 1 & 1 \\
0 & 1 & 2 \\
0 & 0 & 1
\end{array}\right] \cdot\left[\begin{array}{l}
\theta_{0} \\
\theta_{1} \\
\theta_{2}
\end{array}\right]
$$

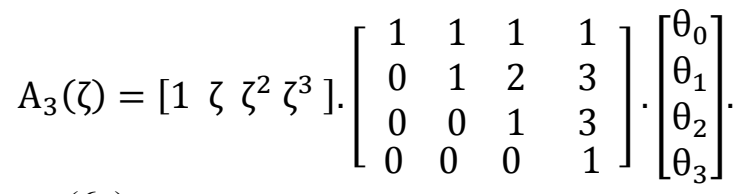

$$
\begin{aligned}
& \mathrm{A}_{4}(\zeta)= \\
& {\left[1 \zeta \zeta^{2} \zeta^{3} \zeta^{4}\right] \cdot\left[\begin{array}{lllll}
1 & 1 & 1 & 1 & 1 \\
0 & 1 & 2 & 3 & 4 \\
0 & 0 & 1 & 3 & 6 \\
0 & 0 & 0 & 1 & 4 \\
0 & 0 & 0 & 0 & 1
\end{array}\right] \cdot\left[\begin{array}{c}
\theta_{0} \\
\theta_{1} \\
\theta_{2} \\
\theta_{3} \\
\theta_{4}
\end{array}\right]}
\end{aligned}
$$

$A_{5}(\zeta)=$

$\left[1 \zeta \zeta^{2} \zeta^{3} \zeta^{4} \zeta^{5}\right] \cdot\left[\begin{array}{cccccc}1 & 1 & 1 & 1 & 1 & 1 \\ 0 & 1 & 2 & 3 & 4 & 5 \\ 0 & 0 & 1 & 3 & 6 & 10 \\ 0 & 0 & 0 & 1 & 4 & 10 \\ 0 & 0 & 0 & 0 & 1 & 5 \\ 0 & 0 & 0 & 0 & 0 & 1\end{array}\right] \cdot\left[\begin{array}{c}\theta_{0} \\ \theta_{1} \\ \theta_{2} \\ \theta_{3} \\ \theta_{4} \\ \theta_{5}\end{array}\right]$

Overall

$$
\begin{aligned}
& \mathrm{A}_{\mathrm{n}}(\zeta)= \\
& {\left[\begin{array}{ccccc}
\delta_{00} & \delta_{01} & \delta_{02} & \cdots & \delta_{0 \mathrm{n}} \\
0 & \delta_{11} & \delta_{12} & \cdots & \delta_{1 \mathrm{n}} \\
0 & 0 & \delta_{22} & \cdots & \delta_{2 \mathrm{n}} \\
\vdots & \vdots & \vdots & \ddots & \\
0 & 0 & 0 & \cdots & \delta_{\mathrm{nn}}
\end{array}\right] \cdot\left[\begin{array}{c}
\theta_{0} \\
\theta_{1} \\
\cdot \\
\cdot \\
\cdot \\
\theta_{\mathrm{n}}
\end{array}\right]}
\end{aligned}
$$

\section{The Solution of the (LVIEs-2k):}

In this section, the (TPs) can be used to determine the (AN) solutions. Remember that the Eq. (1) in the form:

The Eq. (4) can be converted as follows: 


$$
\begin{aligned}
A(\zeta)=k(\zeta)+\alpha & \int_{\substack{\mathrm{a} \\
\subseteq \mathrm{R}}}^{\zeta} \mathrm{G}(\zeta, \tau) \mathrm{A}(\mathrm{8})
\end{aligned}
$$

By using the Eq. (3), let

$A(\zeta) \cong A_{n}(\zeta)$

$=\sum_{\mathrm{r}=0}^{\mathrm{n}} \theta_{\mathrm{r}} Z_{\mathrm{r}}(\zeta)$,

now, substituting the Eq. (9) into the Eq. (8) yields:

$\sum_{\mathrm{r}=0}^{\mathrm{n}} \theta_{\mathrm{r}} \mathrm{Z}_{\mathrm{r}}(\zeta)$

$=\mathrm{k}(\zeta)+\alpha \int_{a}^{\zeta} \mathrm{G}(\zeta, \tau) \sum_{\mathrm{r}=0}^{\mathrm{n}} \theta_{\mathrm{r}} \mathrm{Z}_{\mathrm{r}}(\tau) \mathrm{d} \tau$,

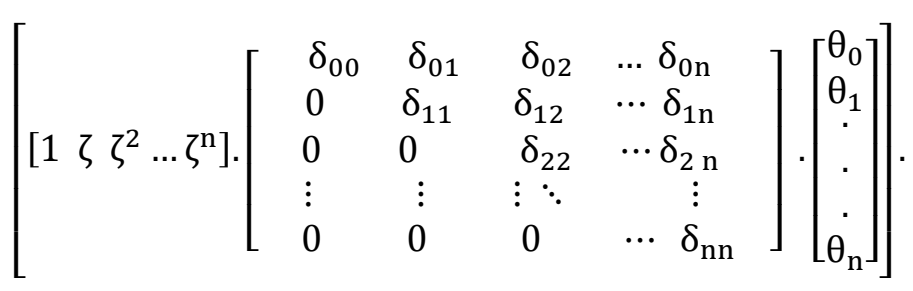

$=\mathrm{k}(\zeta)+\alpha \int_{\mathrm{a}}^{\zeta} \mathrm{G}(\zeta, \tau)\left[\begin{array}{llll}1 & \tau & \tau^{2} & \ldots\end{array} \tau^{\mathrm{n}}\right]$.

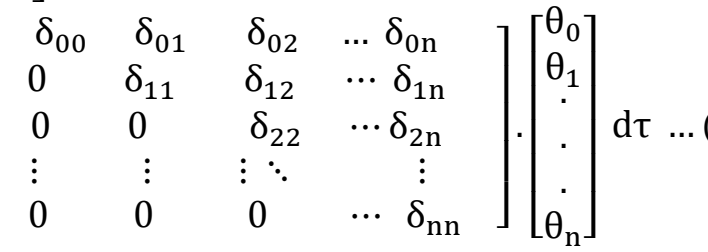

Thus, after computing the integrations (integration methods have been mentioned in the given examples) of the Eq. (12) the unknown coefficients of the (TPs) are found by selecting $\zeta_{\lambda}(\lambda=0,1, \ldots, \mathrm{n})$ from the interval $[0,1]$. Then, get the system of algebraic equations which can be solved by using Gauss elimination method. The unknown coefficients $\left(\theta_{0}, \theta_{1}, \ldots, \theta_{\mathrm{n}}\right)$ have uniquely determined. As a result, by substituting the determined coefficients into the Eq. (3) getting the (AN) solution.

The same procedure can be applied on the Eq. (1a) and (1b) to find the (AN) solutions. by using Eq. (4) then Eq. (10) becomes:

$\left[\begin{array}{llll}\mathrm{Z}_{0}(\zeta) & \mathrm{Z}_{1}(\zeta) & \ldots \mathrm{Z}_{\mathrm{n}}(\zeta)\end{array}\right] \cdot\left[\begin{array}{c}\theta_{0} \\ \theta_{1} \\ \cdot \\ \cdot \\ \theta_{\mathrm{n}}\end{array}\right]$

$=\mathrm{k}(\zeta)$

$+\alpha \int_{a}^{\zeta} G(\zeta, \tau)\left[Z_{0}(\tau) \quad Z_{1}(\tau) \ldots Z_{n}(\tau)\right] \cdot\left[\begin{array}{c}\theta_{0} \\ \theta_{1} \\ \cdot \\ \cdot \\ \theta_{n}\end{array}\right] d \tau, \ldots$

by using the Eq. (5) then the Eq. (11) converted to the general form:

\section{Algorithm of Solution:}

In this section, the steps of the algorithm solution are summarized to find the (AN) solutions for (LVIEs-2K):

Input: $(A(\zeta), k(\zeta), G(\zeta, \tau), a, \zeta)$.

Output: The polynomials of the degree $n$.

Step 1:

Select a degree $\mathrm{n}$ for the (TPs).

$A_{n}(\zeta)=\sum_{r=0}^{n}\left(\begin{array}{l}n \\ r\end{array}\right) \zeta^{r}$

Step 2:

Put the (TPs) into the (LVIEs-2k). 
$\left.\left[\begin{array}{lllll}1 & \zeta & \zeta^{2} & \cdots & \zeta^{\mathrm{n}}\end{array}\right] \cdot\left[\begin{array}{ccccc}\delta_{00} & \delta_{01} & \delta_{02} & \cdots & \delta_{0 \mathrm{n}} \\ 0 & \delta_{11} & \delta_{12} & \cdots & \delta_{1 \mathrm{n}} \\ 0 & 0 & \delta_{22} & \cdots & \delta_{2 \mathrm{n}} \\ \vdots & \vdots & \vdots & & \vdots \\ 0 & 0 & 0 & \cdots & \delta_{\mathrm{nn}}\end{array}\right] \cdot\left[\begin{array}{c}\theta_{0} \\ \theta_{1} \\ \cdot \\ \cdot \\ \cdot \\ \theta_{\mathrm{n}}\end{array}\right]\right]$.

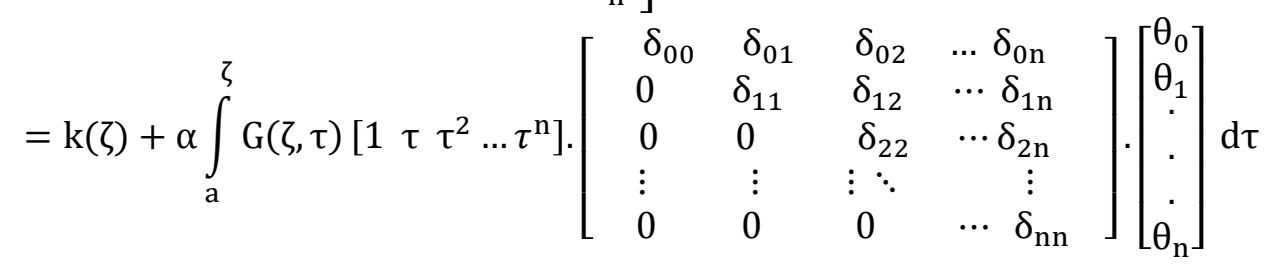

\section{Step 3:}

Computation

$$
\int_{\mathrm{a}}^{\zeta} \mathrm{G}(\zeta, \tau)\left[\begin{array}{lllll}
1 & \tau & \tau^{2} & \ldots & \tau^{\mathrm{n}}
\end{array}\right] \cdot\left[\begin{array}{ccccc}
\delta_{00} & \delta_{01} & \delta_{02} & \cdots & \delta_{0 \mathrm{n}} \\
0 & \delta_{11} & \delta_{12} & \cdots & \delta_{1 \mathrm{n}} \\
0 & 0 & \delta_{22} & \cdots & \delta_{2 \mathrm{n}} \\
\vdots & \vdots & \vdots & & \vdots \\
0 & 0 & 0 & \cdots & \delta_{\mathrm{nn}}
\end{array}\right] \cdot\left[\begin{array}{c}
\theta_{0} \\
\theta_{1} \\
\cdot \\
\cdot \\
\cdot \\
\theta_{\mathrm{n}}
\end{array}\right] \mathrm{d} \tau
$$

Computation

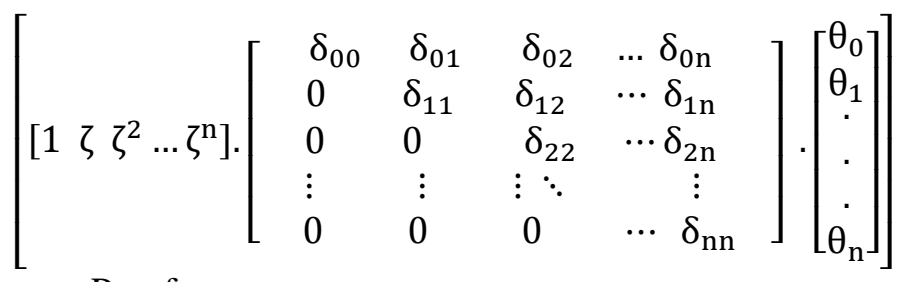

Proof:

\section{Step 4:}

Computation $\theta_{0}, \theta_{1}, \ldots, \theta_{\mathrm{n}}$ where $\zeta_{\lambda} \in[\mathrm{a}, \mathrm{b}], \lambda=$ $0,1,2, \ldots, n$

End.

Note: this algorithm is also suitable for all the other cases.

\section{Accuracy of the Solution:}

To determine the error estimate if the exact (analytic) solution is known, then the absolute error must be the difference between the analytic solution $A(\zeta)$ and the approximate solution $A_{n}(\zeta)$ defined by: $E_{n}(\zeta)=\left|A(\zeta)-A_{n}(\zeta)\right|$.

Definition: With $h$ is a real value function defined and bounded on $[0,1]$, let $A_{n}(h)$ be the polynomial on $[0,1]$, that assigns to $h(\zeta)$ the following value:

$A_{n}(h)=\sum_{r=0}^{n}\left(\begin{array}{l}n \\ r\end{array}\right) \zeta^{r} h\left(\frac{r}{n}\right)$,

where $A_{n}(h)$ is the $n$th Touchard polynomials for

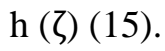

Theorem: For all functions $\mathrm{h}$ in $\mathrm{C}[0,1]$, the sequence of $A_{n}(h)$ converges uniformly to $h$.
The error estimate is presented here which demonstrates the applicability of the presented method. Considering $E=(C[L],\|\|$.$) the Banach$ space of all continuous functions on $\mathrm{L}$.

Suppose that there is a constant $M$ such that $|G(\zeta, \tau)| \leq M$, for all $(\zeta, \tau) \in[0,1]^{2}$. Also, suppose that the linear term satisfies the Lipschitz condition.

Let $A(\zeta)$ be the exact solution and $A_{n}(\zeta)$ be the approximate solution of Eq. (1).

Thus, by taking $\mathrm{n}$ of terms from Touchard polynomials, then:

$$
\begin{aligned}
& \left\|A(\zeta)-A_{n}(\zeta)\right\|= \\
& \max _{\zeta \in L} \mid \mathrm{k}(\zeta)+\alpha \int_{a}^{\zeta} \mathrm{G}(\zeta, \tau) \mathrm{A}(\tau) \mathrm{d} \tau-\mathrm{A}_{\mathrm{n}}(\mathrm{h}(\zeta))- \\
& \alpha \int_{\mathrm{a}}^{\zeta} \mathrm{G}(\zeta, \tau) \mathrm{A}_{\mathrm{n}}(\tau) \mathrm{d} \tau \mid \\
& \quad=\left|\mathrm{k}(\zeta)-\mathrm{A}_{\mathrm{n}}(\mathrm{h}(\zeta))\right|+ \\
& \left|\alpha \int_{\mathrm{a}}^{\zeta} \mathrm{G}(\zeta, \tau)\left(\mathrm{A}(\tau)-\mathrm{A}_{\mathrm{n}}(\tau)\right) \mathrm{d} \tau\right| .
\end{aligned}
$$

Now, by using the above definition and convergence theorem, have:

$\left\|A(\zeta)-A_{n}(\zeta)\right\|=\varepsilon+\alpha \int_{a}^{\zeta}|G(\zeta, \tau)| \mid A(\tau)-$ $A_{\mathrm{n}}(\tau) \mid \mathrm{d} \tau$ 
$A_{n}(\zeta) \mid d \tau$

$$
\leq \varepsilon+\alpha \int_{\mathrm{a}}^{\zeta} \mathrm{M} \max _{\zeta \in \mathrm{L}} \mid \mathrm{A}(\zeta)-
$$

$$
\leq \beta\left\|A(\zeta)-A_{n}(\zeta)\right\|
$$

Then, if $0<\beta<1$, the approximate numerical solution converges to exact solution as $n \rightarrow \infty$ (15). The proof is completed.

\section{Convergence Rate:}

The error defined by the following relation (16):

$\left\|E_{n}\right\|=\left(\int_{0}^{1} E_{n}^{2}(\zeta) d \zeta\right)^{1 / 2} \cong\left(\frac{1}{n} \sum_{\lambda=0}^{n} E_{n}^{2}\left(\zeta_{\lambda}\right)\right)^{1 / 2}$,

where $\left\|E_{n}\right\|$ is an arbitrary vector norm, $E_{n}\left(\zeta_{\lambda}\right)=A(\zeta)-A_{n}\left(\zeta_{\lambda}\right), \quad(\lambda=0,1,2 \ldots n)$. The absolute error $\left|E_{n}\right| \cong\left|A(\zeta)-A_{n}\left(\zeta_{\lambda}\right)\right|$, where $A_{n}\left(\zeta_{\lambda}\right)$ and $A(\zeta)$ are the approximate solutions of the degree $n$ and the exact solution of the (VIEs) respectively.

\section{Illustrative Examples:}

In this section, to show the efficiency and accuracy of the presented numerical method, implement it with comparison of three examples and three examples for test.

Example 1: Consider the (LVIEs-2k) given by (16):

$$
\begin{aligned}
A(\zeta)=\cos (\zeta) & -e^{\zeta} \sin (\zeta) \\
& +\int_{0}^{\zeta} e^{\zeta} A(\tau) d \tau, \quad \zeta \\
& \in[0,1]
\end{aligned}
$$

where $\quad \mathrm{k}(\zeta)=\cos (\zeta)-\mathrm{e}^{\zeta} \sin (\zeta), \alpha=$ $1, G(\zeta, \tau)=e^{\zeta}$, and the exact solution is: $A(\zeta)=$ $\cos (\zeta)$.

By applying an algorithm of the presented method which is described in the above section for this example in case $n=2$, have:

$$
\begin{aligned}
& {\left[\begin{array}{lll}
1 & \zeta & \zeta^{2}
\end{array}\right] \cdot\left[\begin{array}{lll}
1 & 1 & 1 \\
0 & 1 & 2 \\
0 & 0 & 1
\end{array}\right] \cdot\left[\begin{array}{l}
\theta_{0} \\
\theta_{1} \\
\theta_{2}
\end{array}\right]} \\
& =\cos (\zeta)-\mathrm{e}^{\zeta} \sin (\zeta) \\
& +\int_{0}^{\zeta} \mathrm{e}^{\zeta}\left[\begin{array}{lll}
1 & \tau & \tau^{2}
\end{array}\right] \cdot\left[\begin{array}{lll}
1 & 1 & 1 \\
0 & 1 & 2 \\
0 & 0 & 1
\end{array}\right] \cdot\left[\begin{array}{l}
\theta_{0} \\
\theta_{1} \\
\theta_{2}
\end{array}\right] \mathrm{d} \tau,
\end{aligned}
$$

this can be rewritten as:

$$
\begin{aligned}
\theta_{0}+\theta_{1}(1+\zeta) & +\theta_{2}\left(1+2 \zeta+\zeta^{2}\right) \\
& =\cos (\zeta)-\mathrm{e}^{\zeta} \sin (\zeta) \\
& +\int_{0}^{\zeta} \mathrm{e}^{\zeta}\left(\theta_{0}+\theta_{1}(1+\tau)+\theta_{2}(1\right. \\
& \left.\left.+2 \tau+\tau^{2}\right)\right) \mathrm{d} \tau
\end{aligned}
$$

Thus, after computing the integrations by using the power rule, choosing $\zeta_{\lambda}(\lambda=0,1,2)$ in the interval $[0,1]$, getting three equations:

$$
\begin{aligned}
\frac{32046999}{36028797} \theta_{0}+ & \frac{70901578}{72057594} \theta_{1}+\frac{11760478}{10808639} \theta_{2} \\
& =\frac{79684096}{90071993} \\
\frac{68069157}{90071993} \theta_{0} & +\frac{83883271}{90071993} \theta_{1}+\frac{25751724}{22517998} \theta_{2} \\
& =\frac{66420106}{90071993} \\
\frac{26798325}{45035996} \theta_{0} & +\frac{75146947}{90071993} \theta_{1}+\frac{51854731}{45035996} \theta_{2} \\
& =\frac{31323995}{56294995}
\end{aligned}
$$

Solve these equations by using Gauss elimination, and then the coefficients are:

$$
\begin{aligned}
& \theta_{0}=0.51318, \quad \theta_{1}=0.97648, \quad \theta_{2}= \\
& -0.48950
\end{aligned}
$$

Substituting into the Eq. (3), the (AN) solution is:

$$
\begin{gathered}
\mathrm{A}_{2}(\zeta)=0.5132 \mathrm{Z}_{0}(\zeta)+0.9765 \mathrm{Z}_{1}(\zeta) \\
-0.4895 \mathrm{Z}_{2}(\zeta)
\end{gathered}
$$

Now, by applying the same previous procedure for $\mathrm{n}=3,4,5,6$ and 7 , then the (AN) solutions are respectively:

$$
\begin{gathered}
\mathrm{A}_{3}(\zeta)=0.4420 \mathrm{Z}_{0}+1.1550 \mathrm{Z}_{1}-0.6385 \mathrm{Z}_{2} \\
+0.0413 \mathrm{Z}_{3} . \\
\mathrm{A}_{4}(\zeta)=0.5378 \mathrm{Z}_{0}+0.8464 \mathrm{Z}_{1}-0.2665 \mathrm{Z}_{2} \\
-0.1574 \mathrm{Z}_{3}+0.0397 \mathrm{Z}_{4} .
\end{gathered}
$$

$\mathrm{A}_{5}(\zeta)=0.5481 \mathrm{Z}_{0}+0.8061 \mathrm{Z}_{1}-0.2040 \mathrm{Z}_{2}-$ $0.2057 \mathrm{Z}_{3}+0.0583 \mathrm{Z}_{4}-0.0029 \mathrm{Z}_{5}$

$\mathrm{A}_{6}(\zeta)=$

$0.5407 \mathrm{Z}_{0}+0.8395 \mathrm{Z}_{1}-$

$0.2665 \mathrm{Z}_{2}-0.1434 \mathrm{Z}_{3}+0.0235 \mathrm{Z}_{4}+0.0075 \mathrm{Z}_{5}$ $-0.0013 \mathrm{Z}_{6}$.

$A_{7}(\zeta)=$

$0.5399 \mathrm{Z}_{0}+0.8438 \mathrm{Z}_{1}-0.2760 \mathrm{Z}_{2}-0.1320 \mathrm{Z}_{3}+$ $0.0153 \mathrm{Z}_{4}+0.0110 \mathrm{Z}_{5}-0.0021 \mathrm{Z}_{6}+0.0000863 \mathrm{Z}_{7}$ Table 1 shows the comparison of the results. Also Fig. 1 shows the exact and the (AN) solutions for $n$ $=7$ 
Table 1. The Comparison Errors $\left\|E_{n}\right\|$ of Example 1.

\begin{tabular}{ccc}
\hline $\mathrm{n}$ & \multicolumn{2}{c}{$\left\|\mathrm{E}_{\mathrm{n}}\right\|$ of example 1 } \\
\cline { 2 - 3 } 2 & Method of $(16)$ & Present Method \\
3 & $1.95950 \mathrm{E}-03$ & $2.8568 \mathrm{E}-02$ \\
4 & $2.27437 \mathrm{E}-04$ & $7.8726 \mathrm{E}-03$ \\
5 & $6.17039 \mathrm{E}-06$ & $2.7592 \mathrm{E}-04$ \\
6 & $5.39633 \mathrm{E}-07$ & $3.5448 \mathrm{E}-05$ \\
7 & $1.09152 \mathrm{E}-08$ & $7.0276 \mathrm{E}-07$ \\
\hline
\end{tabular}

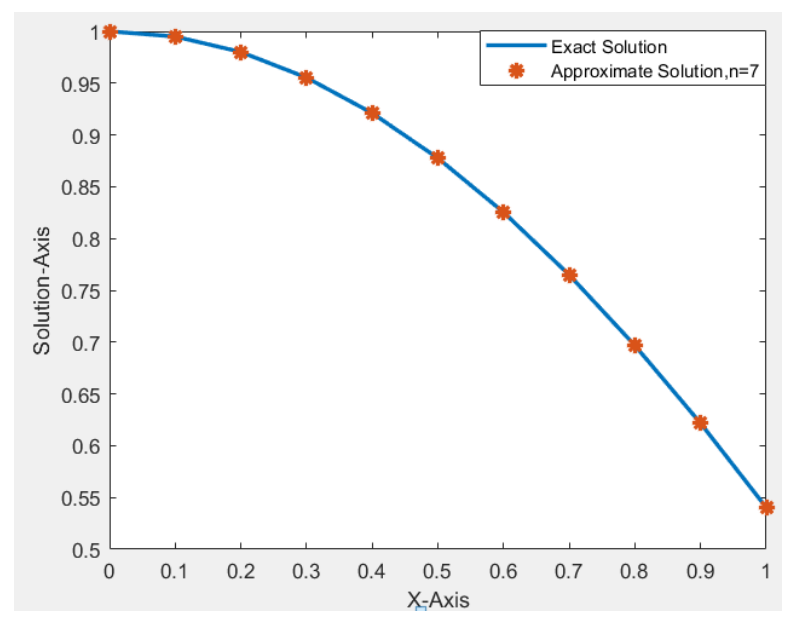

Figure 1. Results of Example 1, Exact Solution and the (AN) Solution for $n=7$.

Example 2: Consider the (LVIEs-1k) given by (16):

$$
\int_{0}^{\zeta} \mathrm{e}^{(\zeta-\tau)} \mathrm{A}(\tau) \mathrm{d} \tau=\sin (\zeta), \quad \zeta \in[0,1]
$$

where $\mathrm{k}(\zeta)=\sin (\zeta), \alpha=1, \mathrm{G}(\zeta, \tau)=\exp ^{(\zeta-\tau)}$, the exact solution is: $A(\zeta)=\cos (\zeta)-\sin (\zeta)$.

By applying the same algorithm for this example and computing the integrations by using the integration of natural exponential function and the integration by using parts formula, and then the comparison results are shown in Table 2. Also Fig. 2 shows the exact and the (AN) solution for $\mathrm{n}=7$.

Table 2. The Comparison Errors $\left\|E_{n}\right\|$ of Example 2.

\begin{tabular}{ccc}
\hline $\mathrm{n}$ & \multicolumn{2}{c}{$\left\|\mathrm{E}_{\mathrm{n}}\right\|$ of Example 2 } \\
\cline { 2 - 3 } 2 & Method of $(16)$ & Present Method \\
3 & $5.06401 \mathrm{E}-02$ & $1.1940 \mathrm{E}-01$ \\
4 & $2.07936 \mathrm{E}-03$ & $6.7191 \mathrm{E}-03$ \\
5 & $6.14967 \mathrm{E}-04$ & $1.2897 \mathrm{E}-03$ \\
6 & $1.42477 \mathrm{E}-04$ & $3.3775 \mathrm{E}-05$ \\
7 & $5.41139 \mathrm{E}-05$ & $3.5964 \mathrm{E}-06$ \\
\hline
\end{tabular}

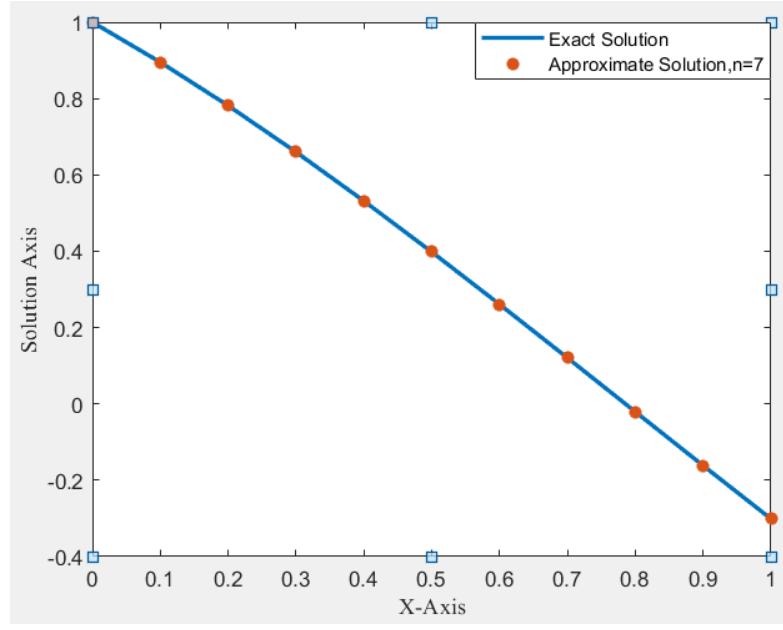

Figure 2. Results of Example 2, Exact Solution and (AN) Solution for $n=7$.

Example 3: Consider Abel's singular (LVIEs-1k) given by (16):

$$
\begin{aligned}
\int_{0}^{\zeta} \frac{1}{\sqrt{\zeta-\tau}} \mathrm{A}(\tau) \mathrm{d} \tau & \\
& =\frac{2 \sqrt{\zeta}}{105}\left(105-56 \zeta^{2}+48 \zeta^{3}\right), \quad \zeta \\
& \in[0,1]
\end{aligned}
$$

where $\quad \mathrm{k}(\zeta)=\frac{2 \sqrt{\zeta}}{105}\left(105-56 \zeta^{2}+48 \zeta^{3}\right), \alpha=$ $1, \mathrm{G}(\zeta, \tau)=\frac{1}{\sqrt{\zeta-\tau}}$, the exact solution is: $\mathrm{A}(\zeta)=$ $\zeta^{3}-\zeta^{2}+1$

Now, by applying the previous algorithm for $n=2$, $3, \ldots, 7$ and computing the integrations by using parts formula, and then the (AN) solutions are respectively:

$$
\begin{aligned}
\mathrm{A}_{2}(\zeta)=0.5925 \mathrm{Z}_{0}+0.8960 \mathrm{Z}_{1}-0.4857 \mathrm{Z}_{2} \\
\mathrm{~A}_{3}(\zeta)=-\mathrm{Z}_{0}+5 \mathrm{Z}_{1}-4 \mathrm{Z}_{2}+\mathrm{Z}_{3} \\
\mathrm{~A}_{4}(\zeta)=-\mathrm{Z}_{0}+5 \mathrm{Z}_{1}-4 \mathrm{Z}_{2}+\mathrm{Z}_{3}+1.8885 \mathrm{E} \\
-13 \mathrm{Z}_{4} . \\
\mathrm{A}_{5}(\zeta)=-\mathrm{Z}_{0}+5 \mathrm{Z}_{1}-4 \mathrm{Z}_{2}+\mathrm{Z}_{3} \\
+5.3841 \mathrm{E}-12 \mathrm{Z}_{4}-8.1480 \mathrm{E} \\
-13 \mathrm{Z}_{5} \\
\mathrm{~A}_{6}(\zeta)=-\mathrm{Z}_{0}+5 \mathrm{Z}_{1}-4 \mathrm{Z}_{2}+\mathrm{Z}_{3} \\
+5.3139 \mathrm{E}-11 \mathrm{Z}_{4}-1.5306 \mathrm{E} \\
-11 \mathrm{Z}_{5}+1.8254 \mathrm{E}-12 \mathrm{Z}_{6} \\
\mathrm{~A}_{7}(\zeta)=-\mathrm{Z}_{0}+5 \mathrm{Z}_{1}-4 \mathrm{Z}_{2}+\mathrm{Z}_{3} \\
+5.3139 \mathrm{E}-11 \mathrm{Z}_{4}-1.5306 \mathrm{E} \\
-11 \mathrm{Z}_{5}+1.8254 \mathrm{E}-12 \mathrm{Z}_{6}+0
\end{aligned}
$$

The comparison results are shown in Table 3. Also Fig. 3 shows the exact and the (AN) solution for $\mathrm{n}=$ 7. 
Table 3. The Comparison Errors $\left\|E_{n}\right\|$ of Example 3.

\begin{tabular}{ccc}
\hline $\mathrm{n}$ & \multicolumn{2}{c}{$\left\|\mathrm{E}_{\mathrm{n}}\right\|$ of Example 3 } \\
\cline { 2 - 3 } 2 & Method of $(16)$ & Present Method \\
3 & $6.39819 \mathrm{E}-02$ & $5.1892 \mathrm{E}-01$ \\
4 & $2.42366 \mathrm{E}-02$ & $5.4209 \mathrm{E}-07$ \\
5 & $3.26226 \mathrm{E}-03$ & $4.6947 \mathrm{E}-07$ \\
6 & $1.15000 \mathrm{E}-03$ & $4.1990 \mathrm{E}-07$ \\
7 & $1.85755 \mathrm{E}-04$ & $3.8332 \mathrm{E}-07$ \\
\hline
\end{tabular}

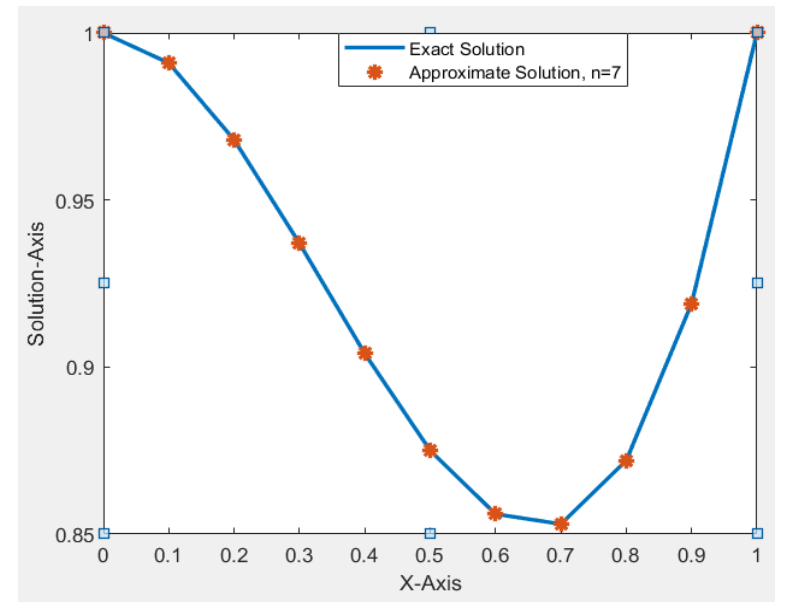

Figure 3. Results of Example 3, Exact Solution and the (AN) Solution for $n=7$.

Example 4: Now consider the (LVIEs-2k) given by (4):

$$
\begin{aligned}
A(\zeta)=5 \zeta^{3}-\zeta^{5} & \zeta \\
& +\int_{0}^{\zeta} \tau \mathrm{A}(\tau) \mathrm{d} \tau, \quad \zeta \\
\in[0,1] & , \quad \zeta
\end{aligned}
$$

where $\mathrm{k}(\zeta)=5 \zeta^{3}-\zeta^{5}, \alpha=1, \mathrm{G}(\zeta, \tau)=\tau$, and the exact solution is: $\mathrm{A}(\zeta)=5 \zeta^{3}$

Now, by applying the same algorithm for $n=2,3, \ldots$, 7 and computing the integrations by using the power rule, then, the (AN) solutions are respectively:

$\mathrm{A}_{2}(\zeta)=3.5836 \mathrm{Z}_{0}-6.5560 \mathrm{Z}_{1}+3.0025 \mathrm{Z}_{2}$.

$A_{3}(\zeta)=-5 Z_{0}+15 Z_{1}-15 Z_{2}+5 Z_{3}$.

$\mathrm{A}_{4}(\zeta)=-5 \mathrm{Z}_{0}+15 \mathrm{Z}_{1}-15 \mathrm{Z}_{2}+5 \mathrm{Z}_{3}+0$.

$A_{5}(\zeta)=-5 Z_{0}+15 Z_{1}-15 Z_{2}+5 Z_{3}+0+0$.

$A_{6}(\zeta)=-5 Z_{0}+15 Z_{1}-15 Z_{2}+5 Z_{3}+0+0$ +0 .

$\mathrm{A}_{7}(\zeta)=-5 \mathrm{Z}_{0}+15 \mathrm{Z}_{1}-15 \mathrm{Z}_{2}+5 \mathrm{Z}_{3}+0+0+$ $0+0$.

The computed errors are shown in Table 4. Also Fig. 4 shows the exact and the (AN) solution for $n=$ 7.

Example 5: Consider another (LVIEs-2k) given by (4):
$\mathrm{A}(\zeta)=\zeta+\zeta^{4}+\frac{1}{2} \zeta^{2}+\frac{1}{5} \zeta^{5}-\int_{0}^{\zeta} \mathrm{A}(\tau) \mathrm{d} \tau, \quad \zeta \in[0,1]$

where $\mathrm{k}(\zeta)=\zeta+\zeta^{4}+\frac{1}{2} \zeta^{2}+\frac{1}{5} \zeta^{5}, \alpha=$

$1, \mathrm{G}(\zeta, \tau)=1$, the exact solution is: $\mathrm{A}(\zeta)=\zeta+\zeta^{4}$,

Now, by applying the same algorithm for $n=2$,

$3, \ldots, 7$ and computing the integrations by using the power rule, then the (AN) solutions are respectively:

$\mathrm{A}_{2}(\zeta)=-0.68955 \mathrm{Z}_{0}+0.44500 \mathrm{Z}_{1}$ $+0.24796 \mathrm{Z}_{2}$.

$\mathrm{A}_{3}(\zeta)=-2.3957 \mathrm{Z}_{0}+4.7342 \mathrm{Z}_{1}-3.3374 \mathrm{Z}_{2}$ $+0.99666 Z_{3}$.

$\mathrm{A}_{4}(\zeta)=-2.8232 \mathrm{e}-13 \mathrm{Z}_{0}-3 \mathrm{Z}_{1}+6 \mathrm{Z}_{2}-4 \mathrm{Z}_{3}$ $+\mathrm{Z}_{4}$.

$A_{5}(\zeta)=-1.5673 E-12 Z_{0}-3 Z_{1}+6 Z_{2}-4 Z_{3}$ $+\mathrm{Z}_{4}+3.5774 \mathrm{E}-13 \mathrm{Z}_{5}$.

$\mathrm{A}_{6}(\zeta)=-7.9198 \mathrm{E}-12 \mathrm{Z}_{0}-3 \mathrm{Z}_{1}+6 \mathrm{Z}_{2}-$ $4 \mathrm{Z}_{3}+\mathrm{Z}_{4}+9.3103 \mathrm{E}-12 \mathrm{Z}_{5}-1.1059 \mathrm{E}-12 \mathrm{Z}_{6}$ $\mathrm{A}_{7}(\zeta)=-4.5151 \mathrm{E}-11 \mathrm{Z}_{0}-3 \mathrm{Z}_{1}+6 \mathrm{Z}_{2}-$ $4 \mathrm{Z}_{3}+\mathrm{Z}_{4}+1.6560 \mathrm{E}-10 \mathrm{Z}_{5}-3.8466 \mathrm{E}-$ $11 \mathrm{Z}_{6}+3.8146 \mathrm{E}-12 \mathrm{Z}_{7}$.

The computed errors are shown in Table 4. Also Fig. 5 shows the exact and the (AN) solution for $\mathrm{n}=7$.

Table 4. The Computed Errors $\left\|E_{n}\right\|$ of the Present Method of Examples 4 and 5.

\begin{tabular}{lcc}
\hline $\mathrm{n}$ & \multicolumn{2}{c}{$\left\|\mathrm{E}_{\mathrm{n}}\right\|$ of Examples 4 and 5 } \\
\cline { 2 - 3 } 2 & Example 4 & Example 5 \\
3 & $2.3158 \mathrm{E}+00$ & $7.1586 \mathrm{E}-01$ \\
4 & $2.7465 \mathrm{E}-06$ & $2.0767 \mathrm{E}-01$ \\
5 & $2.3786 \mathrm{E}-06$ & $1.2191 \mathrm{E}-06$ \\
6 & $2.1274 \mathrm{E}-06$ & $1.0904 \mathrm{E}-06$ \\
7 & $1.9421 \mathrm{E}-06$ & $9.9539 \mathrm{E}-07$ \\
\hline
\end{tabular}

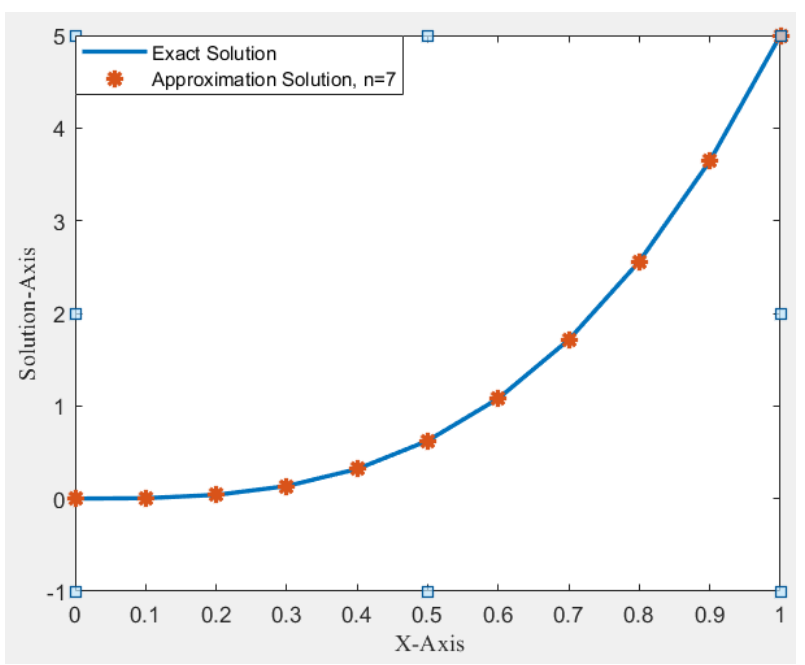

Figure 4. Results of Example 4, Exact Solution and the (AN) Solution for $n=7$. 


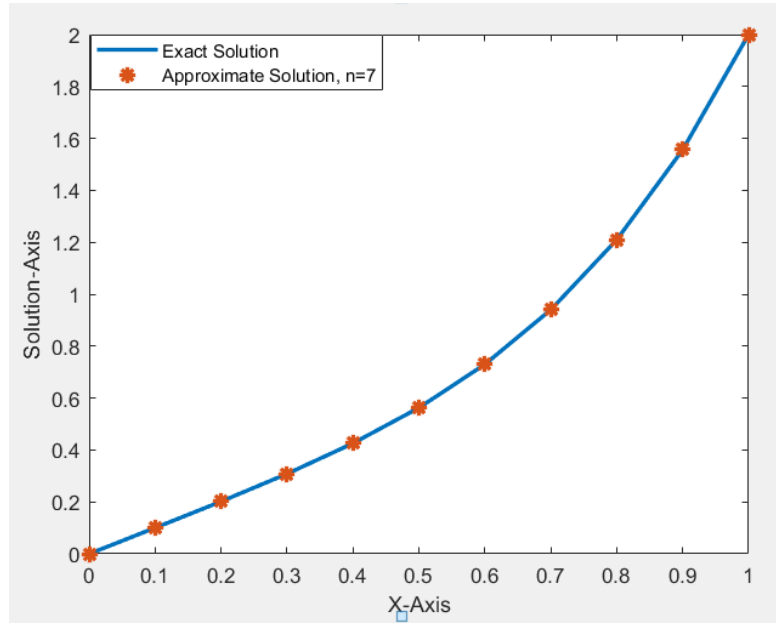

Figure 5. Results of Example 5, Exact Solution and the (AN) Solution for $n=7$.
$\mathrm{A}(\zeta)=\zeta+\int_{0}^{\zeta}(\tau-\zeta) \mathrm{A}(\tau) \mathrm{d} \tau \quad, \quad \zeta \in[0,1]$

where $\mathrm{k}(\zeta)=\zeta, \alpha=1, \mathrm{G}(\zeta, \tau)=(\tau-\zeta)$, the exact solution is: $A(\zeta)=\sin (\zeta)$

By applying the same algorithm, then for $n=4$ and 5 , and computing the integrations by using the power rule, then the (AN) solutions are respectively:

$\mathrm{A}_{4}(\zeta)=-0.81208 \mathrm{Z}_{0}+0.42624 \mathrm{Z}_{1}+0.59631 \mathrm{Z}_{2}$ $-0.22275 \mathrm{Z}_{3}+0.012293 \mathrm{Z}_{4}$.

$A_{5}(\zeta)=-0.84023 Z_{0}+0.53582 Z_{1}+0.42621 Z_{2}$

$-0.091119 \mathrm{Z}_{3}-0.038484 \mathrm{Z}_{4}$

$+0.0078118 \mathrm{Z}_{5}$.

The comparison results are shown in Table 5. Also Fig. 6 shows the exact and the (AN) solution for $\mathrm{n}=5$.

Example 6: Consider the (LVIEs-2k) of the convolution type given by (4):

Table 5.The Comparison of Absolute Errors $\left|E_{n}\right|$ of the Presented Method of Example 6.

\begin{tabular}{|c|c|c|c|c|c|}
\hline \multirow{2}{*}{$\zeta$} & \multirow{2}{*}{$\begin{array}{c}\text { Exact } \\
\text { Solution }\end{array}$} & $\begin{array}{c}\text { Approximate } \\
\text { Solution }\end{array}$ & Absolute Error & $\begin{array}{c}\text { Approximate } \\
\text { Solution }\end{array}$ & Absolute Error \\
\hline 0 & 0 & $9.6606 \mathrm{E}-06$ & $9.6606 \mathrm{E}-06$ & $3.1106 \mathrm{E}-07$ & $3.1106 \mathrm{E}-07$ \\
\hline 0.1 & $9.9833 \mathrm{E}-02$ & $9.9833 \mathrm{E}-02$ & 0 & $9.9833 \mathrm{E}-02$ & 0 \\
\hline 0.2 & $1.9867 \mathrm{E}-01$ & $1.9867 \mathrm{E}-01$ & 0 & $1.9867 \mathrm{E}-01$ & 0 \\
\hline 0.3 & $2.9552 \mathrm{E}-01$ & $2.9552 \mathrm{E}-01$ & 0 & $2.9552 \mathrm{E}-01$ & 0 \\
\hline 0.4 & $3.8942 \mathrm{E}-01$ & $3.8942 \mathrm{E}-01$ & 0 & $3.8942 \mathrm{E}-01$ & 0 \\
\hline 0.5 & $4.7943 \mathrm{E}-01$ & $4.7943 \mathrm{E}-01$ & 0 & $4.7943 \mathrm{E}-01$ & 0 \\
\hline 0.6 & $5.6464 \mathrm{E}-01$ & $5.6463 \mathrm{E}-01$ & $1.0000 \mathrm{E}-06$ & $5.6464 \mathrm{E}-01$ & 0 \\
\hline 0.7 & $6.4422 \mathrm{E}-01$ & $6.4416 \mathrm{E}-01$ & $6.0000 \mathrm{E}-05$ & $6.4422 \mathrm{E}-01$ & 0 \\
\hline 0.8 & $7.1736 \mathrm{E}-01$ & $7.1716 \mathrm{E}-01$ & $2.0000 \mathrm{E}-04$ & $7.1736 \mathrm{E}-01$ & 0 \\
\hline 0.9 & $7.8333 \mathrm{E}-01$ & $7.8281 \mathrm{E}-01$ & $5.2000 \mathrm{E}-04$ & $7.8334 \mathrm{E}-01$ & $1.0000 \mathrm{E}-05$ \\
\hline 1.0 & $8.4147 \mathrm{E}-01$ & $8.4033 \mathrm{E}-01$ & $1.1400 \mathrm{E}-03$ & $8.4151 \mathrm{E}-01$ & $4.0000 \mathrm{E}-05$ \\
\hline
\end{tabular}

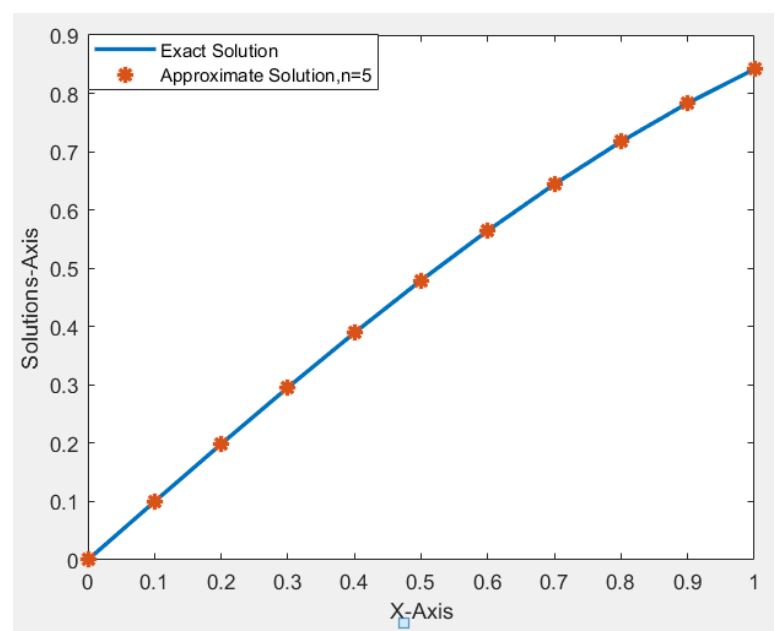

Figure 6. Results of Example 6, Exact Solution and the (AN) Solution for $n=5$.

Note: the symbols $\left\|E_{n}\right\|$ and $\left|E_{n}\right|$ were defined in the section of convergence rate.

\section{Conclusions:}

The prior knowledge have used in a new concept, by using the Touchard polynomials to construct matrices and get functions of the approximation. These functions are used to find the approximate numerical solutions for several kinds of mathematical equations: The integral equations, in particular (LVIEs) of all kinds. The results showed that the present method for solving (LVIEs) of the first and the second kind, also with singular kernel is very effective and their accuracy is high. The Tables and Figs. support this claim. The results indicated that when the polynomial degree $n$ increases, the error decreases rapidly. Moreover, the presented method has been tested by six examples, and approximate numerical results have been compared with one another method. Consequently, the comparison is compatible with it or better. 


\section{Author's declaration:}

- Conflicts of Interest: None.

- I hereby confirm that all the Figures and Tables in the manuscript are mine. Besides, the Figures and images, which are not mine, have been given the permission for re-publication attached with the manuscript.

- Ethical Clearance: The project was approved by the local ethical committee in Wasit University .

\section{References:}

1. Eleonora M, Antonia V. Stability and Boundless of Numerical Approximations to Volterra Integral Equations. APPL NUMER MATH. 2017 June; 116: 230-237

2. Hashmi M. S, Khan N, Iqbal S. Numerical Solutions of Weakly Singular Volterra Integral Equations Using the Optimal Homotopy Asymptotic Method. COMPUT MATH APPL. 2012; 64 (2012) : 15671574.

3. Abdul J. J. Introduction to Integral Equations with Applications. New York: MARCEL DEKKER; 1985. 73- 74 P.

4. Abdul-Majid W. Linear and Nonlinear Integral Equations Methods and Applications. Heidelberg Dordrecht London New-York: Springer; 2011. 35-36 p.

5. Mohamed M.S, Gepreel K. A, Al-Malki F. A, AlHumyani M. Approximate solutions of the generalized Abel's integral equations using the extension Khan's homotopy analysis transformation method. J APPL MATH. 2015. 9 pages. Available from: https://doi.org/10.1155/2015/357861

6. Muftahov I, Tynda A, Sidorov D. Numerical Solution of Volterra Integral Equations of the First Kind with Discontinuous Kernel. J COMPUT APPL MATH. 2017 Mar 15; 313(15): 119-128
7. Marjan U, Muhammad T. On the Approximation of Volterra Integral Equations with Highly Oscillatory Bessel Kernels via Laplace Transform and Quadrature. AEJ. 2019; 58(2019): 413-417.

8. Hashmi M. S, Khan N, Iqbal S. Numerical Solutions of Weakly Singular Volterra Integral Equations Using the Optimal Homotopy Asymptotic Method. COMPUT MATH APPL.2012 Sept; 64 (6):15671574.

9. Can H, Martin S. Spectral Galerkin Methods for a Weakly Singular Volterra Integral Equation of the Second Kind. IMA JNA. 2017 July; 37(3): 14111436.

10. Xiao-yong Z. A Multistep Legendre Pseudo-Spectral Method for Volterra Integral Equations. APPL MATH COMPUT. 2016 Feb 1; 274: 480-494.

11. Nazir A, Usman M, Mohyud-Din ST.Touchard Polynomials Method for Integral Equations. Int. J. Modern Theo. Physics, 2014; 3(1): 74-89.

12. Paris R. B. The Asymptotes of the Touchard Polynomials: a uniform approximation. Math. Æterna. 2016 Jun 28; 6(5): 765-779.

13. Miloud M, Mohammed S. M. Touchard Polynomials, Partial Bell Polynomials and Polynomials of Binomial Type. Journal of Integer Sequences. 2011 Mar 25; 14(2011): 1-9.

14. Sun ZW, Zagier D. ON A curious Property of Bell Numbers. Bull. Aust. Math. Soc. 2011; 84: 153-158

15. Rani D, Mishra V. Solutions of Volterra Integral and Integro-Differential Equations Using Modified Laplace Adomian Decomposition Method. JAMSI. 2019 June; 15 (1):1-18

16. Maleknejad K, Hashemizadeh E, Ezzati R. A new Approach to the Numerical Solution of Volterra Integral Equations by Using Bernstein's Approximation. Commun Nonlinear Sci Numer Simulat. 2011; 16 (2011): 647-655

\section{الحلول العددية التقريبية لمعادلات فولتيرا التكاملية باستخدام متعددة حدود توشارد}
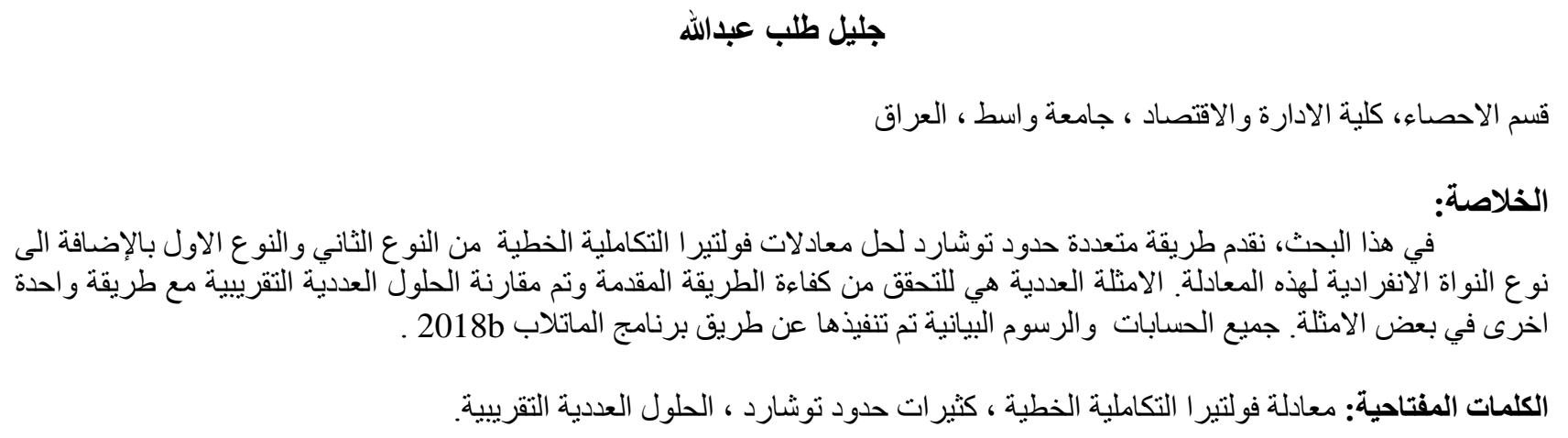